\title{
CIRCUMFERENTIAL ARGON LASER PHOTOCOAGULATION FOR PREVENTION OF RETINAL DETACHMENT
}

\author{
AYALA POLLACK ${ }^{1.2}$. ASHER MILSTEIN ${ }^{\prime}$, MOSHE OLIVER ${ }^{1.2}$ and MIRIAM ZALISH ${ }^{1}$ \\ Reholot. Israel
}

\begin{abstract}
SUMMARY
Circumferential argon laser photocoagulation was administered as preventive treatment for retinal detachment in 53 eyes with extensive areas of lattice degeneration and/or retinal breaks and at least one additional high-risk factor for retinal detachment. Laser burns were applied in several continuous rows $360^{\circ}$ around the peripheral retina at the junction between the posterior border of the lesions and the unaffected retina. The retina posterior to the treated areas remained attached in $\mathbf{5 1}$ eyes $(96.2 \%)$ during a mean follow-up period of 85.8 months (range 6 months to 18 years). The treatment did not affect visual acuity. The only complication was the appearance in 2 eyes $(3.8 \%)$ of a delicate epiretinal membrane which, however, did not require surgical intervention.
\end{abstract}

Prophylactic treatment for retinal detachment is still controversial. ' ${ }^{+}$mainly because of a lack of information about the extent to which peripheral retinal degeneration predisposes the retina towards detachment and whether detachment can be prevented by such treatment. Two recent studies of the natural history of lattice degeneration provide some evidence with regard to the risk of retinal detachment. In a prospective long-term follow-up study of patients with lattice degeneration in phakic non-fellow eyes, Byer ${ }^{3}$ found that clinical retinal detachment occurred in only $1.08 \%$ of patients and in $0.7 \%$ of eyes and advised against preventive laser treatment. Folk et $\mathrm{al}^{4}$ retrospectively analysed the results of prophylactic treatment applied to areas of lattice degeneration and breaks in the fellow eyes of patients with retinal detachment. These eyes were at higher risk than those in the series of Byer, ${ }^{3}$

From: Department of Ophthalmology. Kaplan Hospital. Rehovot: affiliated with ${ }^{2}$ The Hebrew University. Hadassah Medical School. Jerusalem, Israel.

Correspondence to: A. Pollack. MD. Department of Ophthalmology. Kaplan Hospital. 76100 Rehovot. Israel. who studied non-fellow eyes. but the probability of their developing retinal detachment was still low.

Over the last 18 years we have used argon laser photocoagulation as preventive treatment in selected patients considered to be at high risk of developing retinal detachment. We applied the laser marks all around the peripheral retina in order to delimit progression of any retinal detachment that may occur as a result of retinal tearing and retinal holes associated with retinal degeneration. Here we describe the criteria for selection, the lasering technique and its results.

\section{PATIENTS AND METHODS}

Between January 1976 and December 1993, 53 eyes of 44 patients ( 21 males and 23 females) underwent prophylactic argon laser photocoagulation of the peripheral retina in our department. Their ages ranged from 7 to 81 years (mean 43.2 years). The follow-up period ranged from 6 to 216 months (mean 85.8 months).

Indications for treatment were: (1) extensive peripheral retinal degeneration extending over a continuous area of at least 5 clock-hours of lattice degeneration, with or without retinal holes and/or tears, or (2) multiple retinal tears extending over at least three quadrants of the peripheral retina. In addition, candidates for treatment had to have at least one of the following known risk factors for retinal detachment: (a) previous retinal detachment in the fellow eye, (b) a family history of retinal detachment, (c) the presence of an inherited vitreoretinal disease such as snowflake vitreoretinal degeneration, and (d) previous intracapsular cataract extraction with myopia of 7 dioptres or more.

Photocoagulation treatment was applied with an argon laser beam of $300-500 \mu \mathrm{m}$ spot size, $0.1-0.2$ seconds duration and a power level of approximately 400 $600 \mathrm{~mW}$. also adjusted to cause whitening of the retina. Laser burns were applied to the peripheral retina at the

Eve (1994) 8, 419-422 C) 1994 Royal College of Ophthalmologists 
border between normal and pathological retina. Three to six rows, less than one-quarter burn width apart, were applied posteriorly to the areas of retinal pathology and parallel to the ora serrata (Fig. 1). Eyes in which lesions were located at the equator and/or posterior to it and did not reach the ora serrata were given two or three rows of laser burns around the lesion including its anterior border, in addition to the burns applied at the posterior border of the lesion (Fig. 2).

\section{CASE REPORTS}

Two representative patients are described in the following case reports.

\section{Case 1}

A 36-year-old man was referred for cataract surgery in the left eye. Examination revealed a visual acuity of $6 / 12$ with -10.0 spherical equivalent in the right eye and $6 / 120$ with -10.0 in the left eye. Fundal examination disclosed typical findings of snowflake vitreoretinal degeneration in both eyes, ${ }^{5}$ with swirling fibrillar degeneration of the vitreous, liquefaction of the vitreous gel, white with pressure and snowflake degeneration in the peripheral retina, retinal pigmentation, retinal breaks and sheathing of retinal vessels. The left eye underwent extracapsular cataract extraction, which was complicated by a tear in the posterior capsule and vitreous loss. Six months later retinal detachment occurred in the right phakic eye; in spite of two operations the retina remained detached. Three other family members (an uncle, a sister and a nephew) had also experienced retinal detachment in one eye with unsuccessful surgical results.

In view of the family history, the poor surgical outcome in the phakic eye, and the high incidence of retinal detachment with poor surgical prognosis known to occur in patients with snowflake vitreoretinal degeneration ${ }^{5}$ we performed prophylactic argon laser photocoagulation treatment of the peripheral retina in the left eye. ${ }^{6}$ The

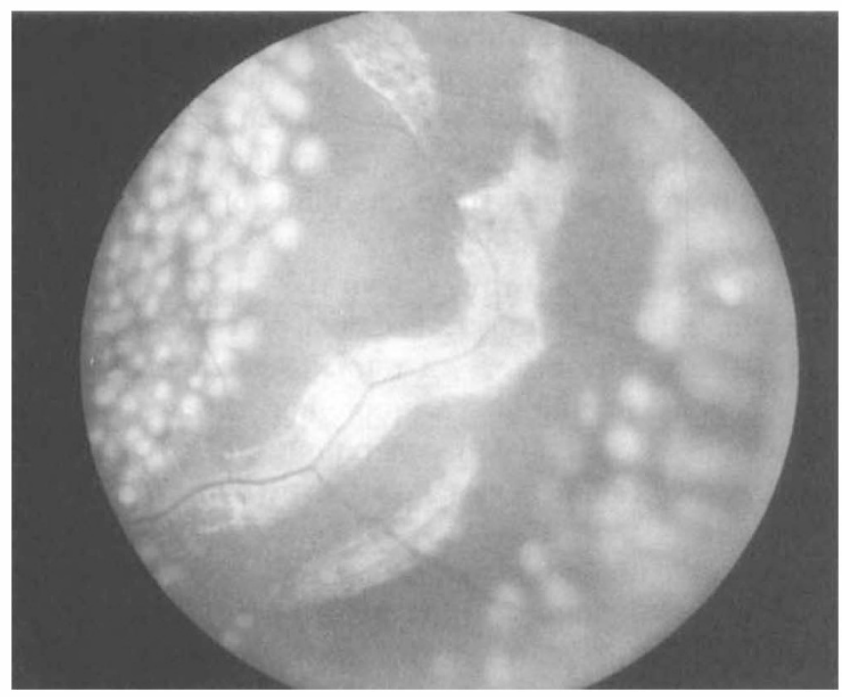

Fig. 1. Ophthalmoscopic appearance of the peripheral retina in a case immediately following laser treatment posterior and anterior to lattice lesions. patient has now been followed for 18 years. In the left eye the retina is attached and the vision is $20 / 50$, with moderate myopic chorioretinal degeneration of the macula.

\section{Case 2}

A 12-year-old boy had been followed since infancy in our department because of retinopathy of prematurity in a cicatricial stage. At the age of 11 years he developed retinal detachment in the right eye. Eye examination prior to surgery revealed a visual acuity of $6 / 30$ with -4.0 spherical equivalent in the right eye and $6 / 12$ with -5.0 in the left. Biomicroscopic examination of the anterior segment was unremarkable. Funduscopy of the right eye disclosed cicatricial retinopathy of prematurity at the peripheral retina and extensive areas of lattice degeneration with multiple retinal holes. The retina showed a detachment of $270^{\circ}$ extending over the temporal and lower half. In the left eye funduscopy disclosed cicatricial retinopathy of prematurity and extensive areas of lattice degeneration extending all around the peripheral retina.

The right eye underwent successful surgery for retinal detachment and the post-operative visual acuity was $6 / 12$. During the first year of post-operative follow-up the right eye remained stable, but the left eye showed progressive changes, with new foci of lattice areas forming a second row of lattice degeneration with new multiple retinal holes. Fifteen months after surgery in the right eye, preventive argon laser photocoagulation was applied in the left eye all around the peripheral retina. After 4 years the vision is 6/12 in both eyes and both retinas are attached.

\section{RESULTS}

The distributions of high-risk factors and refractive errors among the eyes in this series are summarised in Tables I and II.

Of the 53 treated eyes, the retina remained attached in $49(92.4 \%)$; the other $4(7.6 \%)$ subsequently developed retinal detachment. In 2 of these 4 eyes, detachment

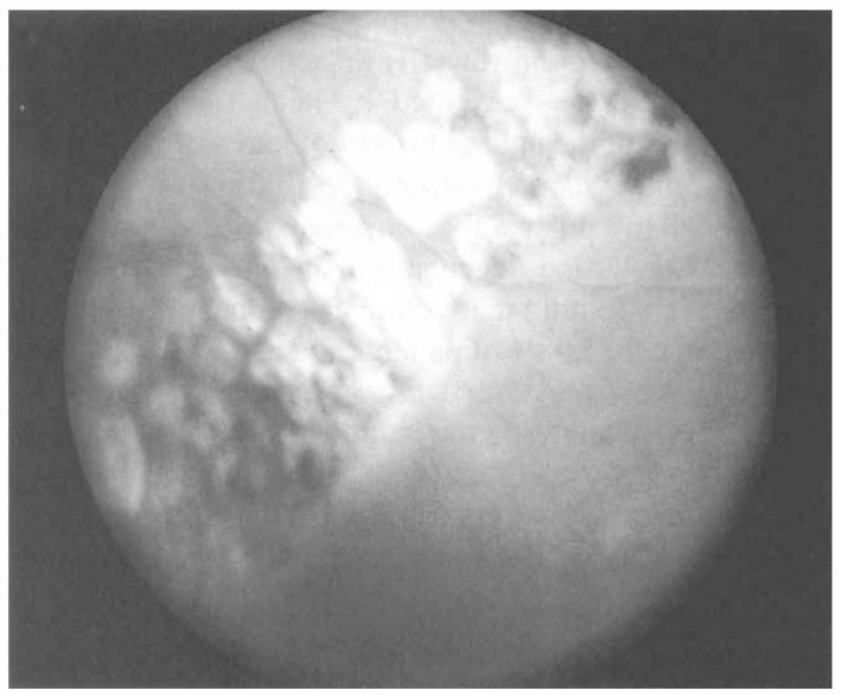

Fig. 2. Scarred laser lesions located posterior to the peripheral detached retina. 
Table I. Risk factors associated with retinal detachment"

\begin{tabular}{lc}
\hline Risk factor & No. of eyes \\
\hline Retinal detachment in the fellow eye & 17 \\
Family history of retinal detachment & 11 \\
Myopia greater than 7 dioptres & 26 \\
Vitreoretinal degeneration & 12 \\
Intracapsular cataract extraction & 19 \\
\hline
\end{tabular}

${ }^{a}$ More than one risk factor was present in 28 eyes.

Table II. Distribution of refractive errors ${ }^{i}$

\begin{tabular}{lcc}
\hline $\begin{array}{c}\leqslant 0 \text { dioptre } \\
\text { (no. of eyes) }\end{array}$ & $\begin{array}{c}0 \text { to }-6.75 \text { dioptre } \\
\text { (no. of eyes) }\end{array}$ & $\begin{array}{c}\leqslant-7.00 \text { dioptre } \\
\text { (no. of eyes) }\end{array}$ \\
\hline 9 & 18 & 26 \\
\hline an admission to the study. &
\end{tabular}

occurred anteriorly to the photocoagulation scars and required no further treatment as it did not progress posteriorly during a mean follow-up period of 81.5 months (Fig. 2). In the other 2 eyes retinal detachment developed because of a macular hole in association with high myopia.

Two eyes $(3.8 \%)$ developed epiretinal membranes. In 1 patient this was diagnosed 3.5 years after treatment, and in the other 2 months after treatment. In the latter patient, however, similar findings were also seen in the fellow untreated eye. In each case the epiretinal membrane was delicate and did not cause marked distortion of blood vessels, and the vision decreased by only 1 line on the Snellen chart. Surgical removal was therefore not indicated. No other complications were noted.

Visual acuity was unaffected by the laser treatment in 51 eyes $(96.2 \%)$, including the 2 eyes with macular holes in which the vision before and after treatment was counting fingers because of myopic chorioretinal degeneration in the macular area. The vision remained unchanged after repairing the retinal detachment. The eyes that showed visual loss after treatment were the same two that developed epiretinal membranes; their visual acuity decreased by 1 Snellen chart line only.

\section{DISCUSSION}

According to Byer, ${ }^{3}$ lattice degeneration by itself is insufficient indication for treatment aimed at prevention of retinal detachment. However, in patients with retinal holes, tears and/or detachments in the fellow eye, the risk of detachment is much higher. ${ }^{4}$ As pointed out by Boniuk et al. ${ }^{1}$ and McPherson et al., ${ }^{2}$ the risk in these eyes can be reduced by prophylactic treatment. Although Folk et al. ${ }^{4}$ reached a similar conclusion, they nevertheless queried the justification for treating 100 eyes to prevent retinal detachments over a 7 year period. They emphasised, however, that they had excluded patients with hereditary retinopathies, who may be at increased risk of detachment, and that in such selected cases treatment should be considered.

We agree with those authors who believe that prophylactic treatment of eyes with isolated foci of lattice degeneration is not justified. However, we feel that it should be considered in eyes at high risk of developing retinal detachment, ${ }^{7}$ and particularly in eyes with vitreoretinal degeneration syndromes, which are especially vulnerable to retinal detachment, often with poor surgical results. ${ }^{8-10}$ This particular high-risk group is not common: for example, during a follow-up period of 18 years we saw only 53 patients with severe vitreoretinal pathology. Nevertheless, this group of patients raises a serious dilemma for the physician who has to decide whether or not to apply prophylactic treatment. Our policy is to perform such treatment.

We preferred to use argon laser photocoagulation. Cryopexy, which was considered the preferred mode of therapy, ${ }^{10}$ has recently been implicated in the enhancement of proliferative vitreoretinopathy. ${ }^{11}$ Since the vitreoretinal pathology in all our patients was very extensive, we applied laser burns all around the peripheral retina as well as around each individual lesion. According to Folk, lasering is more effective when applied to all areas of lattice degeneration and breaks than when applied only to some of them.

Following treatment, only 4 eyes $(7.6 \%)$ developed retinal detachment during a mean follow-up period of 85.8 months (range 6 months to 18 years). In 2 of these eyes, however, the cause of detachment was not peripheral retinal degeneration but a macular hole. Because this developed more than a year after photocoagulation, and because both affected eyes had a high degree of myopia, it is doubtful whether the pathology in the macular area can be linked to the peripheral retinal pathology or to the prophylactic treatment.

In the other 2 eyes, detachment was induced by pathology of the peripheral retina but was confined to the area anterior to the laser burns, as though the laser scars had halted its progression towards the posterior pole (Fig. 2). Since an aim of the treatment was to limit the extent of any peripheral retinal detachment that might subsequently occur, and thus to preserve visual function, these 2 cases of anterior detachment can be viewed as examples of successful treatment.

As regards the other 49 eyes in which the retina remained attached after treatment, obviously one cannot know how many would have developed retinal detachment if they had been left untreated. However, since all of these eyes were in the high-risk category, it seems reasonable to assume that retinal detachment would have occurred in at least some of them during the follow-up period. For example, in the subgroup of 12 patients who are members of a family with hereditary vitreoretinal degeneration, retinal detachment is expected to occur in between $30 \%$ and $50 \%$ of affected members. ${ }^{9.10}$ The question then arises as to whether this assumption justifies our decision to apply preventive laser treatment in all these patients. In view of the results (i.e. continued attachment of the posterior retina in $96.2 \%$ of cases), and considering the low rate of complications as well as their apparently non-deleterious character, we believe that the answer is 'yes'. 
The way in which laser photocoagulation prevents retinal detachment is not well understood. One possibility is that circumferential lasering may act in the same way as focal treatment of isolated lattice degeneration. ${ }^{+}$It is possible that the boundary of laser burns at the border of pathological and normal retina, produced by the application of laser photocoagulation $360^{\circ}$ around the peripheral retina, acts like a new posterior ora serrata, and results in relatively balanced vitreoretinal tractional forces posterior to the lasered area. In our opinion this technique of reducing vitreous traction is better than a scleral buckling procedure. Circumferential lasering, together with the encircling of individual lesions, may result in: (1) fewer new tears, (2) a lower incidence of retinal detachment from existing or new breaks, and (3) arrested progression of peripheral retinal detachment towards the centre. It should, however, be emphasised that laser treatment in this study was neither intended nor expected to prevent the formation of new peripheral lesions or breaks. The occurrence of new tears in areas that appear clinically normal $^{2.3 .12-15}$ and in prophylactically treated eyes ${ }^{15}$ has been described previously. As pointed out by Folk et al., ${ }^{15}$ the new lesions do not appear to be directly associated with the lasering procedure.

Assessment of the effectiveness of the prophylactic laser treatment in this study is difficult because extensive vitreoretinal pathology occurs only rarely, and therefore the number of candidates for treatment is not only small but also highly variable in terms of age, aetiology and specific retinal features. We are aware that results obtained with such a group may not be conclusive. Nevertheless. we are encouraged by the results of this study showing that laser treatment confines retinal detachment to the anterior retina in high-risk cases.

In conclusion, our experience suggests that complete circumferential peripheral argon laser photocoagulation for prevention of retinal detachment is a safe and reliable procedure, and is associated with an extremely low rate of complications. However, it should be considered only in carefully selected cases characterised by extensive vitreoretinal pathology and additional high-risk factors for the development of retinal detachment.
Key words: Argon laser photocoagulation. High risk, Preventive treatment. Retinal detachment.

\section{REFERENCES}

1. Boniuk I, Okun E. Johnston GP, Arribas N. Xenon photocoagulation vs. cryotherapy in the prevention of retinal detachment. Mod Probl Ophthalmol 1974;12:81-92.

2. McPherson A, O'Malley R, Beltangady SS. Management of the fellow eyes of patients with rhegmatogenous retinal detachment. Ophthalmology 1981:88:922-34.

3. Byer NE. Long-term natural history of lattice degeneration of the retina. Ophthalmology 1989:96:1396-402.

4. Folk JC, Arrindell EL, Klugman MR. The fellow eye of patients with phakic lattice retinal detachment. Ophthalmology 1989:96:72-9.

5. Hirose T, Lee KY, Schepens CL. Snowflake degeneration in hereditary vitreoretinal degeneration. Am J Ophthalmol 1974:77:143-53.

6. Pollack A, Uchenik D, Chemke J, Oliver M. Prophylactic laser photocoagulation in hereditary snowflake vitreoretinal degeneration: a family report. Arch Ophthalmol 1993: 101: $1536-9$.

7. Byer NE. Lattice degeneration of the retina. Surv Ophthalmol 1979:23:213-38.

8. van Balen ATM, Falger ELF. Hereditary hyaloideoretinal degeneration and palatoschisis. Arch Ophthalmol 1970:83: 152-62.

9. Hirose T, Lee KY. Schepens CL. Wagner`s hereditary vitreoretinal degeneration and retinal detachment. Arch Ophthalmol 1973:89:176-85.

10. Maumenee IH. Vitreoretinal degeneration as a sign of generalized connective tissue diseases. Am J Ophthalmol 1979:88:432-49.

11. Campochiaro PA, Maden IH, Vidaurri-Leal J, Glaser BM. Cryotherapy enhances intravitreal dispersion of viable retinal pigment epithelial cells. Arch Ophthalmol 1985;103: 343-6.

12. Schepens CL. The preventive treatment of idiopathic and secondary retinal detachment. In: Acta XVIII Concilium Ophthalmologicum, 1958 (Belgium), vol. 1:1019-27.

13. Delaney WV, Oates RP. Retinal detachment in the second eye. Arch Ophthalmol 1978:96:629-34.

14. Foos RY. Post-oral peripheral retinal tears. Ann Ophthalmol 1974;6:679-87.

15. Folk JC, Bennett SR, Klugman MR. Arrindell EL, Boldt HC. Prophylactic treatment to the fellow eye of patients with phakic lattice retinal detachment: analysis of failures and risks of treatment. Retina 1990:10:165-9. 